\title{
Sistema de indicadores ambientales de la Universidad Nacional (2008-20I I). Aspectos de significancia: electricidad, agua, combustible y teléfono
}

Environmental indicators system in the Universidad

Nacional (2008-20 I I). Environmental aspects

of significance: electricity-water-fuel-phone

David Benavides Ramírez'

Noelia Garita Sánchez ${ }^{2}$

José Carlos Mora Barrantes ${ }^{3}$

Julián Rojas Vargas ${ }^{4}$

Fecha de recepción: 27 de marzo del 2012

Fecha de aprobación: 24 de octubre del 2012

Benavides, D; Garita, N; Mora, J; Rojas, J. Sistemas de indicadores ambientales de la Universidad Nacional (2008-20 I I). Aspectos de significancia: electricidad, agua, combustible y teléfono.

Tecnología en Marcha. Vol. 26, № I. Pág 66-76.

I Químico Industrial, Universidad Nacional, Costa Rica. Tel. 2277-3554, correo electrónico: dbenavid@una.ac.cr

2 Bióloga, Universidad Nacional, Costa Rica. Tel.2277-3554, correo electrónico: ngarita@una.ac.cr

3 Químico Industrial, Universidad Nacional, Costa Rica. Tel. 2277-3403, correo electrónico: jmor@una.ac.cr

4 Biólogo, Universidad Nacional, Costa Rica. Tel. 2277-3554, correo electrónico: jrojasv@una.ac.cr 


\section{Palabras clave}

Sistema de indicadores ambientales, aspectos ambientales de significancia, consumo de electricidad, agua, combustibles, teléfono, gestión ambiental

\section{Resumen}

Como parte de los esfuerzos ambientales llevados a cabo por la Universidad Nacional (UNA) de Costa Rica, incluyendo todas sus dependencias, oficinas, centros y sedes y el Programa UNA Campus Sostenible, nuestra institución ha consolidado en el tiempo un Sistema de Indicadores Ambientales con aspectos de significancia enmarcados en un Programa de Gestión Ambiental Institucional (PGAI), el cual mide el desempeño ambiental de la institución desde 2008, generando iniciativas como la campaña "Únase al ahorro", la cual busca disminuir la facturación de los servicios públicos.

Por medio de la aplicación de esta herramienta de evaluación ambiental, la Dirección de Gestión de Calidad Ambiental (DIGECA-MINAET) y la UNA han establecido una medición del desempeño ambiental de forma estandarizada e integrada en cuatro aspectos ambientales que se mencionan a continuación:

- Consumo eléctrico

- Consumo de agua

- Consumo de combustibles

- Uso del teléfono

Las principales características del modelo que impulsa el PGAI se basan en los conceptos de integración y sostenibilidad, que analizan los aspectos ambientales mencionados y las actividades asociadas para su mitigación y minimización, con el fin de llegar a una ecoeficiencia en la operatividad propia de la Universidad.

Recientemente, la UNA presentó la "Actualización del Plan de Gestión Ambiental Institucional" ante la DIGECA-MINAET, el cual cumple de manera satisfactoria con lo requerido por esta Dirección, según lo establecido en el Decreto Ejecutivo 36499-S-MINAET.

Se debe mencionar que este proceso se ha llevado a cabo gracias a la colaboración y participación activa de toda la comunidad universitaria y que se deben redoblar esfuerzos para continuar con esta visión ambiental propia y pionera de la UNA hacia los nuevos retos ambientales.

\section{Key words}

Environmental indicators system, environmental aspects, consumption: electricity, water, fuel, telephone, environmental management

\begin{abstract}
As part of the environmental efforts taken by the Universidad Nacional (UNA) -Departments, Offices, Research Centers and the "UNA Campus Sostenible Program"- has established over time a Environmental Indicators System with environmental aspects of significance, framed within the "Programa de Gestión Ambiental Institucional" (PGAI). The UNA has been measuring the environmental performance since 2008, including the peripheral campus and generating initiatives such as the campaign "Únase al ahorro", which aims to decrease the bill of public services.
\end{abstract}

Through the implementation of this environmental assessment tool (PGAI), the UNA and the Dirección de Gestión de Calidad Ambiental (DIGECA) -Unit of the Ministry of Environmental of Costa Rica- have established a consumption control of the public services by standardizing and integrating four significant environmental aspects, which are mentioned below:

- Electricity consumption

- Potable water consumption

- Fuel consumption

- Use of Phone sets and its services

The main features of the model that uses the PGAI are based on the concepts of integration and sustainability, which analyzes the environmental aspects mentioned above related to the activities, in order to achieve the minimization of consumptions, to reach an eco-efficiency in the usual processes of the University.

The UNA recently presented to de DIGECA the PGAI Summary (document), and according to the law requirements (Policy No. No. 36499-S-MINAET), the University satisfactorily complies with the averaged information and environmental indicators.

It must be mentioned that this environmental efforts have been developed with the collaboration and active participation of the entire university community, and that we must redouble efforts to continue this environmental vision and embrace the proposal of new environmental challenges. 


\section{Introducción}

La Universidad Nacional de Costa Rica (UNA), por medio del Programa UNA Campus Sostenible, en su Módulo Técnico, desarrolló el Proyecto "Sistema de Indicadores". UNA Campus Sostenible incluye la creación de sistemas de información ambiental para el análisis de los aspectos ambientales de significancia que genera la interacción propia de la comunidad universitaria.

La falta de información consistente y coherente sobre la calidad ambiental de la UNA muchas veces es una de las restricciones para definir prioridades de gestión ambiental, establecer metas de consumo de recursos naturales y acertar en la toma de decisiones.

El establecimiento de sistemas de información de calidad ambiental como este sistema de indicadores y los esquemas de seguimiento a los mismos, denominados Observatorio Ambiental (Dirección de Investigación), Plan de Ahorro de agua, electricidad, combustible y teléfono (Campaña "Únete al Ahorro") y el Programa de Gestión Ambiental Institucional (PGAI), permiten presentar los indicadores numéricos, gráficos y criterios comparativos que ayuden en la planificación del desarrollo sostenible de la Universidad sobre una base suficientemente objetiva, sistematizada y accesible. El sistema de información genera señales precisas a las autoridades ambientales y a la comunidad universitaria, que permiten acelerar la gestión ambiental integral.

Para el seguimiento y la actualización del Sistema de Indicadores, es de suma importancia generar tendencias en el tiempo y el espacio, de modo que se puedan observar los cambios promovidos por una gestión integral en el consumo de los recursos.

El empoderamiento lógico en la gestión ambiental de la institución contempla los actores claves que se describen en la figura I.
Este sistema de indicadores se realizó con base en información de muchos sectores y actores, tomando en cuenta los siguientes aspectos ambientales (figura 2):

\section{Consumo eléctrico}

Se levantó un listado actualizado de los medidores eléctricos que existen en la UNA por medio del pago de los recibos por parte de la Sección Financiera (ver cuadro I). Estos medidores se solicitaron para todas las sedes. Al momento de tener todos los números de medidores, se solicitaron los consumos mensuales, tanto a la Empresa de Servicios Públicos de Heredia (ESPH), como al Instituto Costarricense de Electricidad (ICE), la Compañía Nacional de Fuerza y Luz (CNFL) y otras empresas de servicios eléctricos. Luego se sumaron los datos mensuales para obtener el dato anual y se realizaron los demás cálculos estadísticos (ver figura 3). Se debe mencionar que para el cálculo per cápita se utilizaron los datos de población universitaria (estudiantes y funcionarios), aportados por el Departamento de Registro y Recursos Humanos, respectivamente (ver figura 4).

\section{Consumo de agua}

Se levantó un listado actualizado de los medidores de agua que existen en la UNA por medio del pago de los recibos por parte de la Sección Financiera (ver cuadro 2). Al momento de tener todos los números de medidores, se solicitaron los consumos mensuales, tanto a la ESPH como al Instituto Costarricense de Acueductos y Alcantarillados (AyA). Luego se sumaron los datos mensuales para obtener el dato anual y se realizaron los demás cálculos estadísticos (ver figura 5). Se debe mencionar que para el cálculo per cápita se utilizaron los datos de población universitaria (estudiantes y funcionarios), aportados por el Departamento de Registro y Recursos Humanos, respectivamente (ver figura 6). 


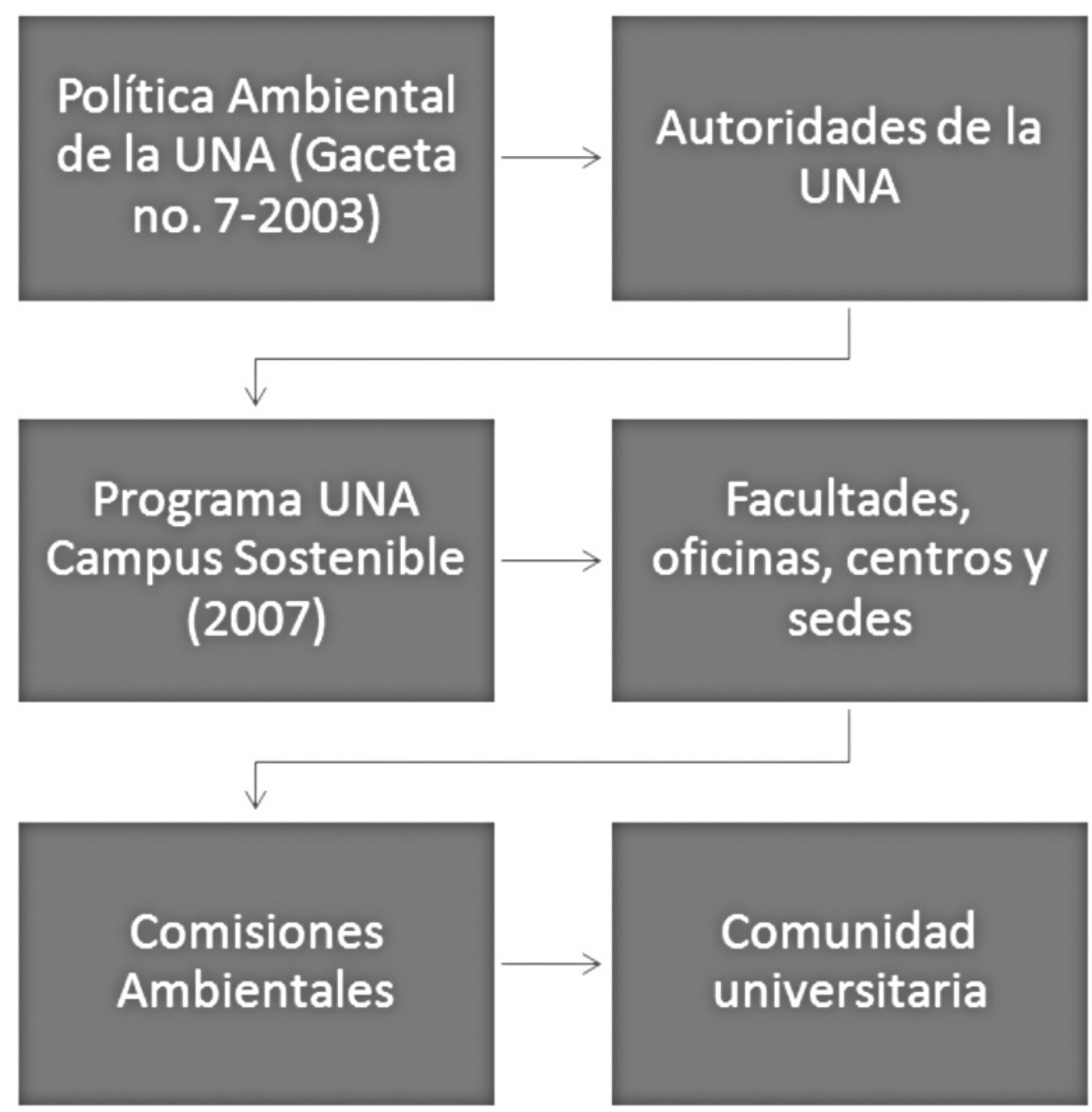

Figura I. Empoderamiento en la UNA de la Gestión Ambiental.Fuente: Elaboración propia.

\section{Consumo de combustibles}

El consumo del combustible fósil se divide en varios sectores: I. El consumo de combustible por flota vehicular de la UNA, 2. El consumo de gas por parte de las sodas y comedores en la UNA y 3. Los viajes aéreos patrocinados por la UNA. Para el consumo de combustible, el dato lo brindó la Sección de Transportes en gasto mensual en colones de toda la flotilla vehicular (ver figura 7). Este dato se dividió por el histórico de precios por litro de combustible de la Refinería Costarricense de Petróleo (RECOPE) para obtener los litros consumidos por mes y luego fue anualizado (ver figura 8).

\section{Uso del teléfono}

Para el periodo 2011-2012, en el marco de la campaña de ahorro de recursos institucionales ("Únete al Ahorro") y la Directriz 017 del MINAET: Eficiencia Energética y el Programa de Gestión Ambiental Institucional, se incluyó como indicador ambiental la facturación por el uso del teléfono.

Se levantó un listado actualizado de los números de teléfono que posee la UNA utilizando el pago de los recibos al ICE a través de la Sección Financiera. Luego se sumaron los datos mensuales para obtener el dato anual y se realizaron los demás cálculos estadísticos (ver figura 9). Se debe mencionar que para el cálculo per cápita se utilizaron los datos de población universitaria que incluye a los funcionarios (administrativos y académicos), aportados por la Sección de Recursos Humanos. 


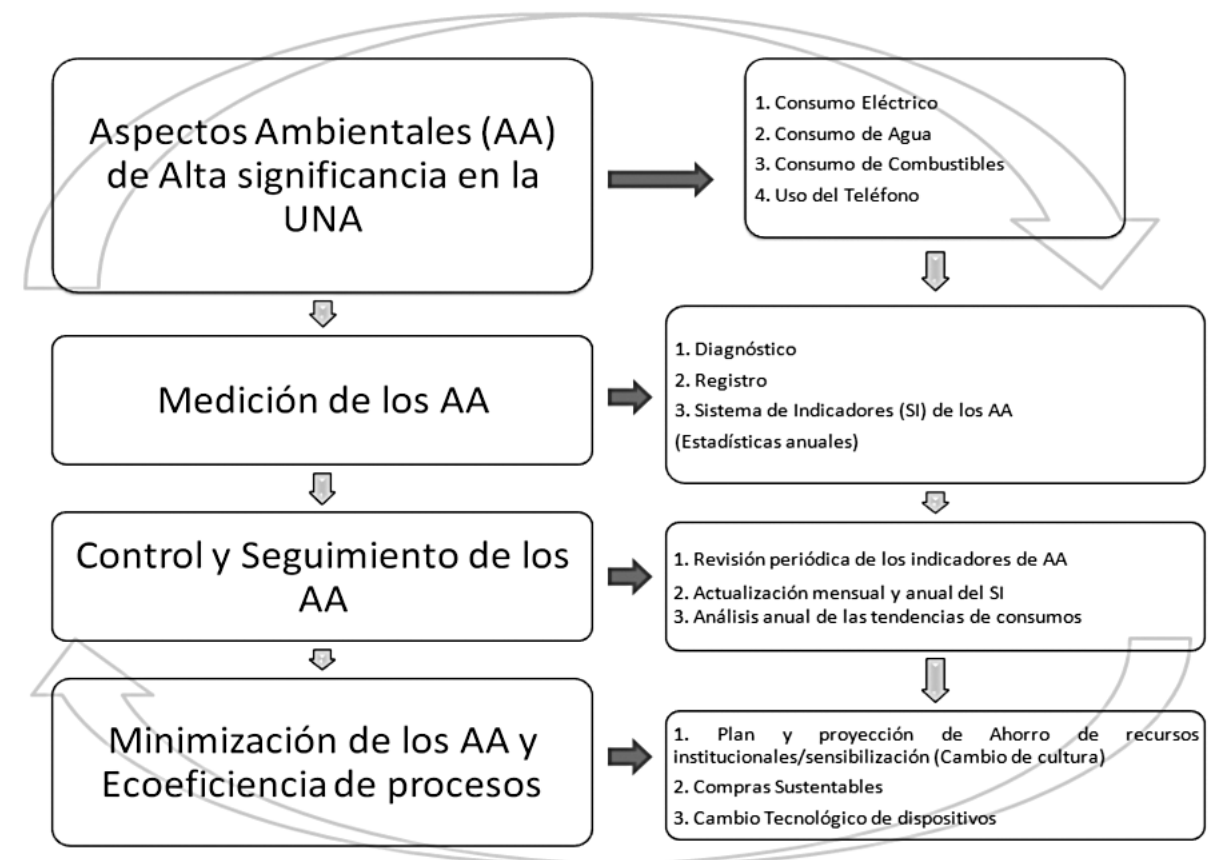

Figura 2. Gestión Ambiental en la UNA fundamentada en el Sistema de Indicadores Ambientales de cuatro Aspectos Ambientales de Significancia. Fuente: Elaboración propia.

Cuadro I. Indicadores de consumo eléctrico

\begin{tabular}{|c|c|c|c|c|c|}
\hline \multirow{3}{*}{ Consumo eléctrico } & UNA 2008 & UNA 2009 & UNA 2010 & UNA 2011 & \\
\hline & \multicolumn{4}{|c|}{ Cantidad } & Unidad \\
\hline & II5 medidores & 124 medidores & 128 medidores & 124 medidores & \\
\hline Consumo anual & 5707383 & 5580932 & $526 \mid 867$ & 5741802 & kWh/año \\
\hline Consumo mensual & 475615 & 465078 & 438489 & 478484 & $\mathrm{kWh} / \mathrm{mes}$ \\
\hline Consumo anual per cápita & 347 & 308 & 261 & 290 & kWh/año/pers. \\
\hline Consumo mensual per cápita & 29 & 26 & 22 & 24 & $\mathrm{kWh} / \mathrm{mes} /$ pers. \\
\hline
\end{tabular}

Nota:Para 201 I la facturación del consumo de electricidad ascendió a \455 600298 colones según la Sección Financiera; para 20 I2, con la campaña "Únase al ahorro", se pretende reducir la facturación en un 2\% (aprox. 9 millones de colones).

Fuente: Sección de Mantenimiento, Sección Financiera, CNFL, ICE, ESPH y UNA Campus Sostenible 2009-20I2. 


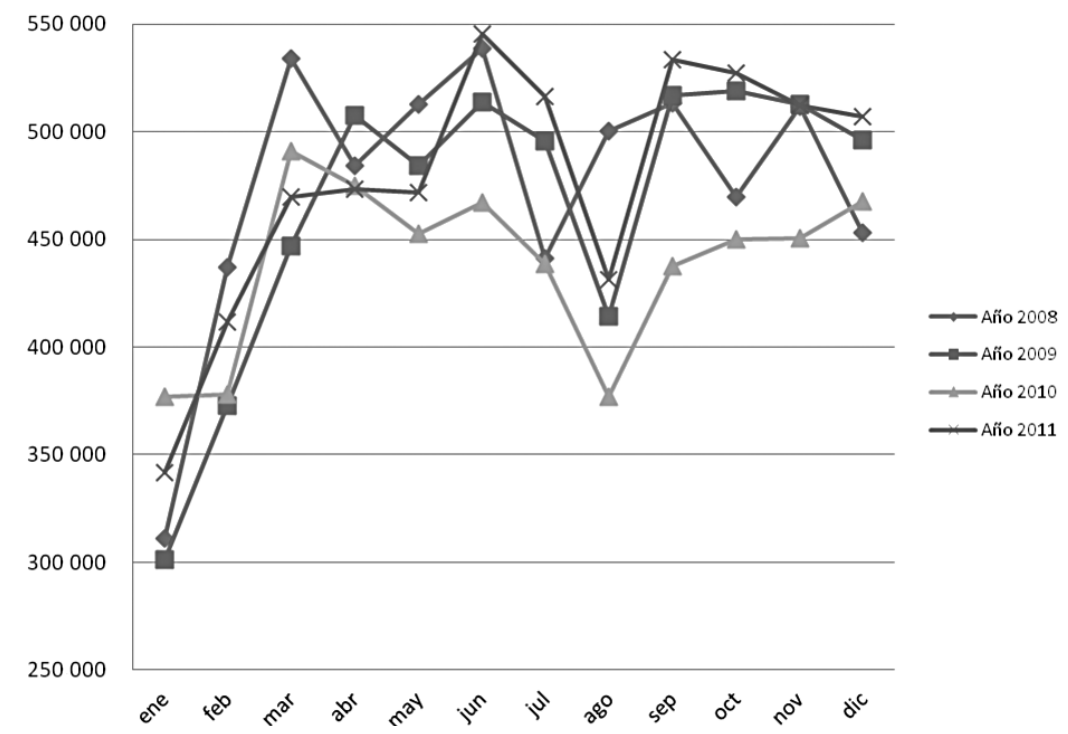

Figura 3. Tendencia mensual de consumo eléctrico en la UNA para 2008-201 I.

Fuente: Sección de Mantenimiento, Sección de Financiero, CNFL, ICE, ESPH y UNA Campus Sostenible 2009-20I 2.

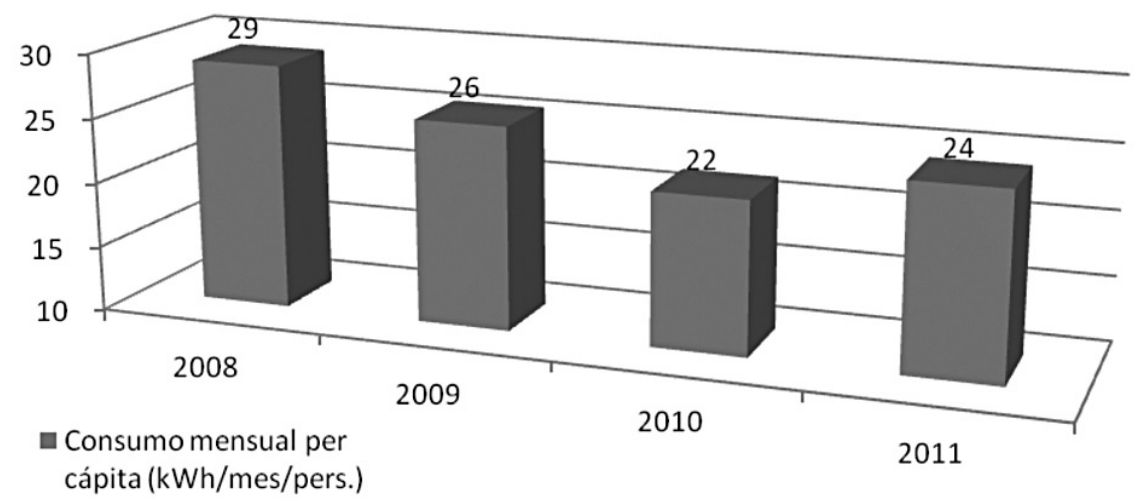

Una persona en promedio (vivienda) consume $75 \mathrm{kWh} / \mathrm{mes}$.

Figura 4. Consumo mensual de electricidad per cápita en la UNA en el periodo 2008-20I I.

Fuente: Sección de Mantenimiento, Sección de Financiero, CNFL, ICE, ESPH y UNA Campus Sostenible 2009-20I2.

Cuadro 2. Indicadores de consumo de agua

\begin{tabular}{|c|c|c|c|c|c|}
\hline \multirow{3}{*}{ Consumo de agua } & UNA 2008 & UNA 2009 & UNA 2010 & UNA 2011 & \\
\hline & \multicolumn{4}{|c|}{ Cantidad } & Unidad \\
\hline & 60 medidores & 78 medidores & 77 medidores & 73 medidores & \\
\hline Consumo anual & 183366 & $22017 \mid$ & 326699 & 329386 & $\mathrm{~m}^{3} / a n ̃ o l$ \\
\hline Consumo mensual & 15281 & 18348 & 27225 & 27449 & $\mathrm{~m}^{3} / \mathrm{mes}$ \\
\hline Consumo anual per cápita & 11 & 12 & 16 & 17 & $\mathrm{~m}^{3} /$ año/pers. \\
\hline \multirow{2}{*}{ Consumo mensual per cápita } & 0,93 & 1,01 & ।,35 & 1,38 & $\mathrm{~m}^{3} / \mathrm{mes} /$ pers. \\
\hline & 929 & 1012 & 1350 & 1385 & L/mes/pers. \\
\hline Consumo diario per cápita & 31 & 34 & 45 & 46 & L²/día/pers. \\
\hline
\end{tabular}

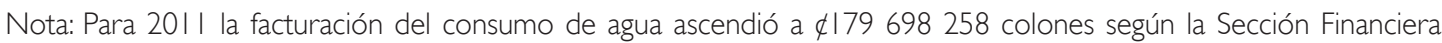
y para 20 I2, con la campaña "Únase al ahorro", se pretende reducir la facturación en un 3\% (aprox. 5.4 millones de colones). Fuente: Sección de Mantenimiento, Sección Financiera, AyA, ESPH y UNA Campus Sostenible 2009-20I2. 


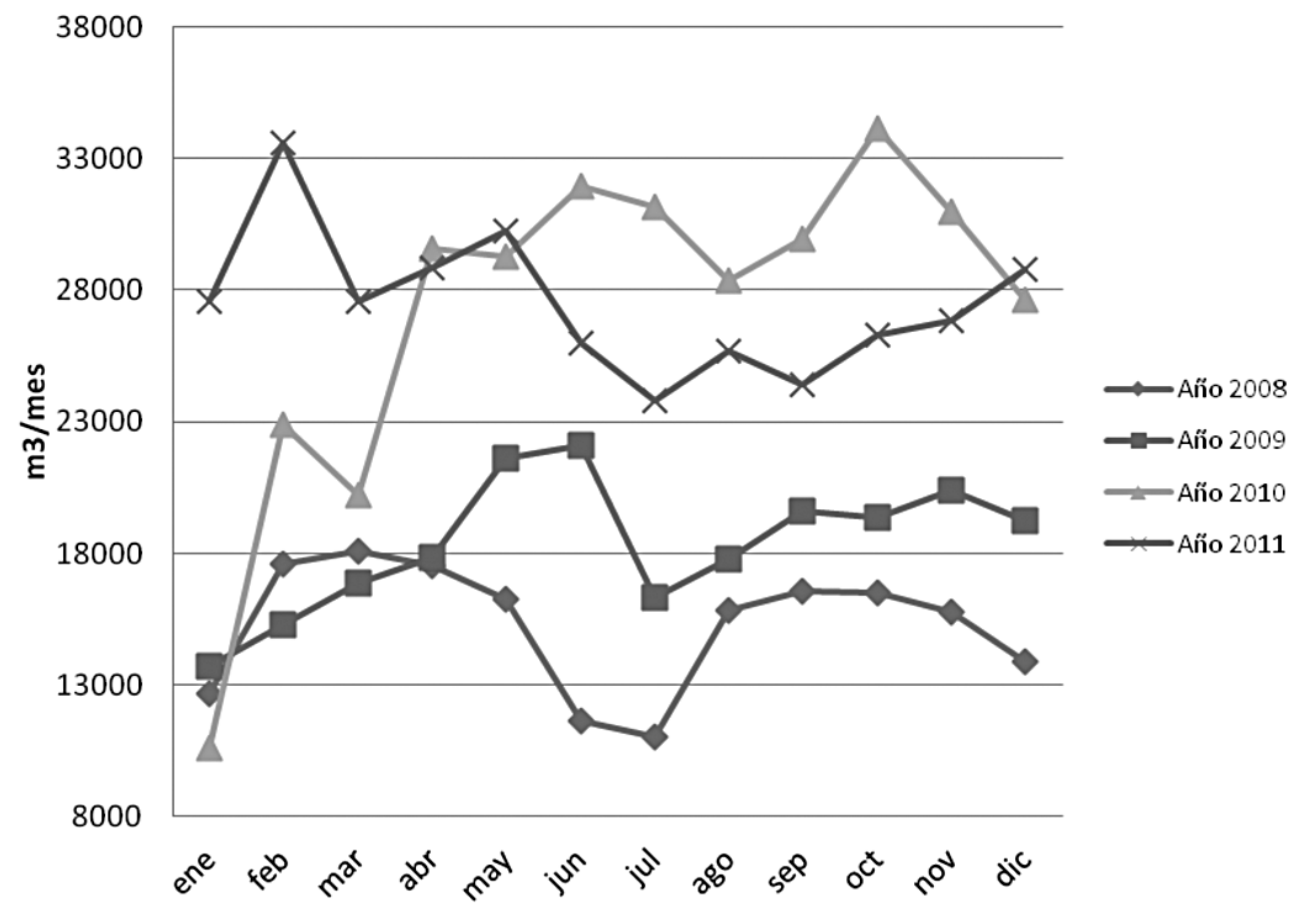

Figura 5. Tendencia mensual de consumo de agua en la UNA para el periodo 2008-201।. Fuente: Sección de Mantenimiento, Sección Financiera, AyA, ESPH y UNA Campus Sostenible 2009-20I2.

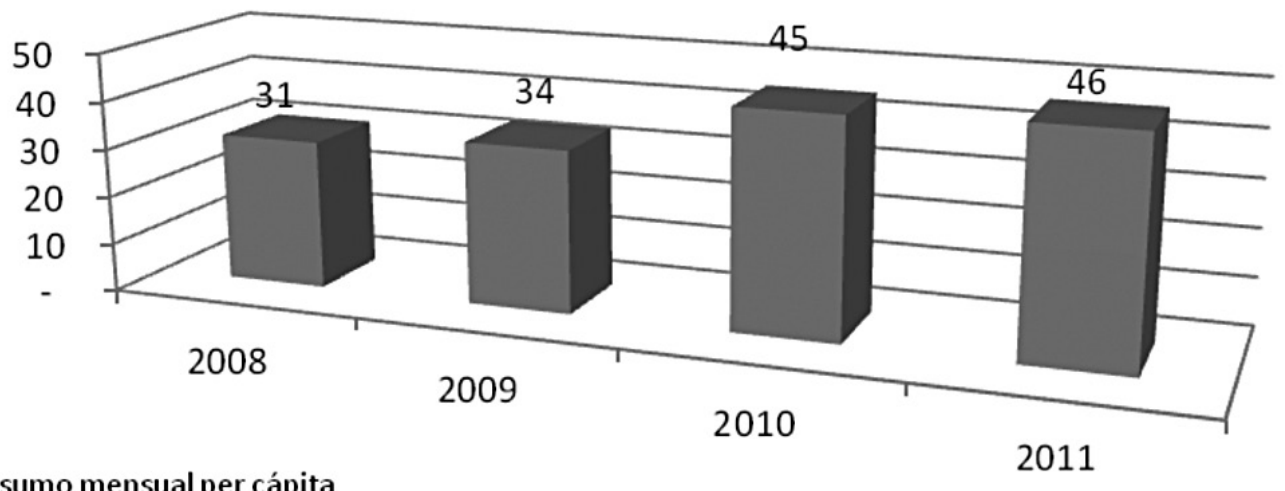

- Consumo mensual per cápita

(L/día/pers.)

Una persona en promedio (vivienda) consume 165 L/día.

Figura 6. Consumo diario de agua per cápita en la UNA en el periodo 2008-20I I. Fuente: Sección de Mantenimiento, Sección de Financiero, AyA, ESPH y UNA Campus Sostenible 2009-2012. 


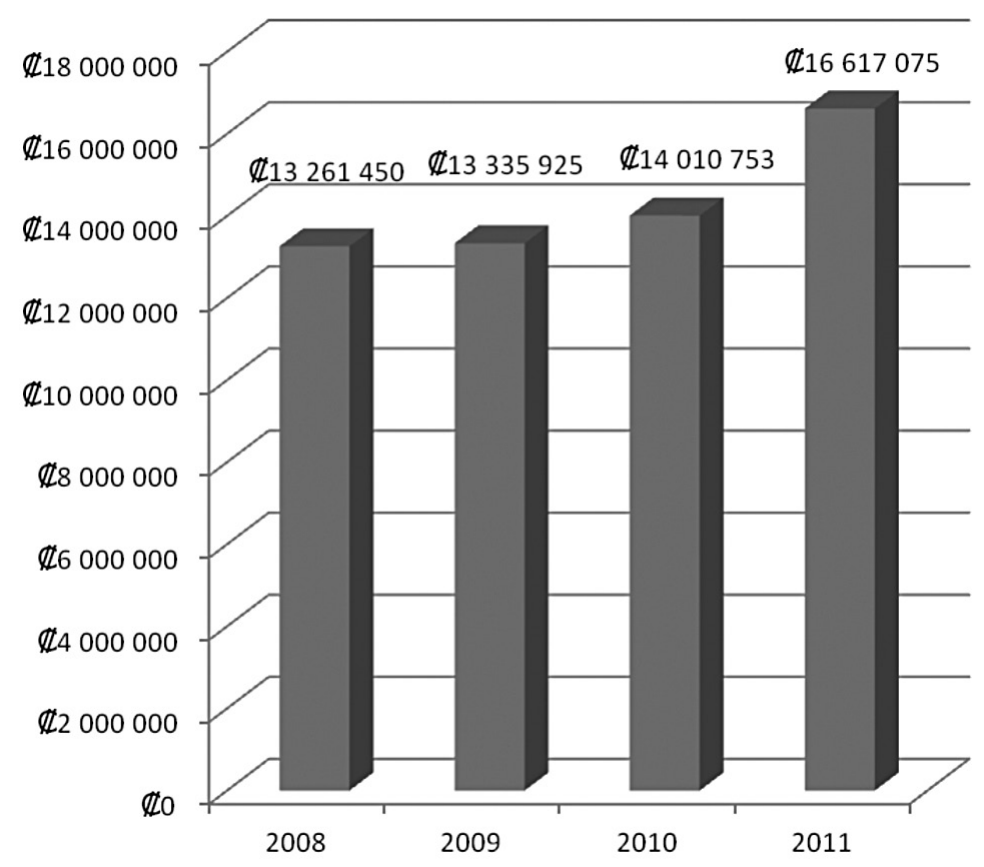

Figura 7. Gasto en colones por mes/promedio por compra de combustible estandarizado a diesel de la flotilla vehicular y plantas eléctricas de la UNA para los años 2008-20 I I. Fuente: Sección Financiera, Sección de Transportes, RECOPE y UNA Campus Sostenible 2009-2012.

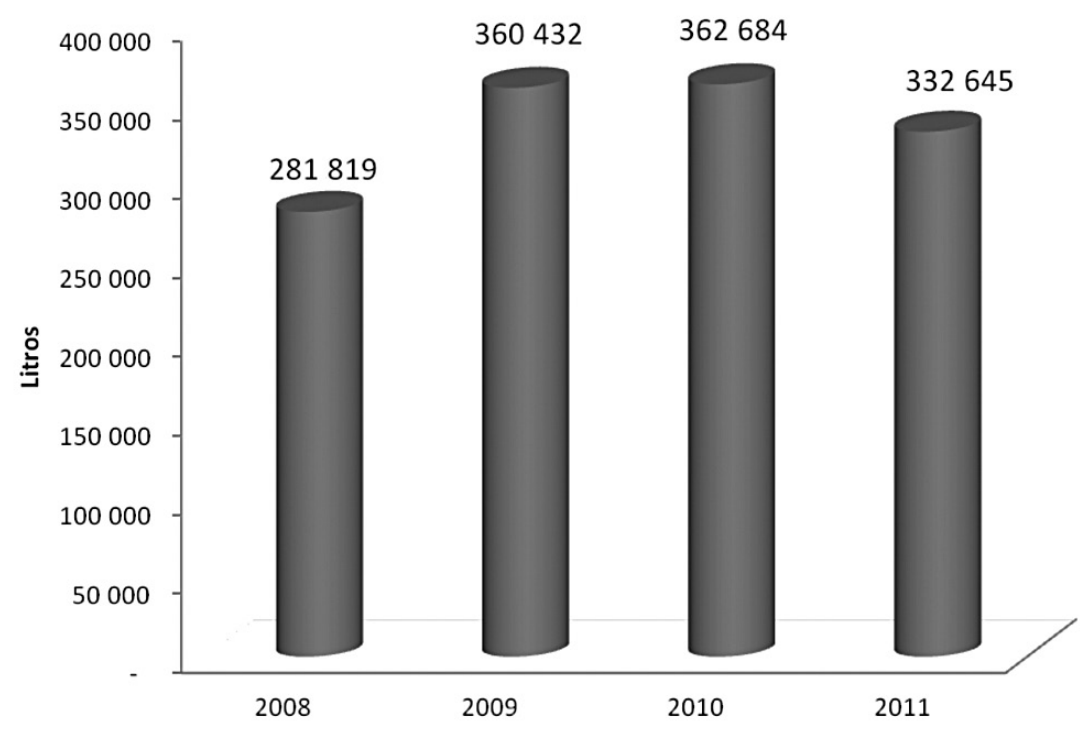

Figura 8. Consumo anual de combustible estandarizado a diesel por la flotilla vehicular y plantas eléctricas de la UNA para los años 2008-20।l.

Fuente: Sección de Financiero, Sección de Transportes, RECOPE y UNA Campus Sostenible 2009-20I2. 


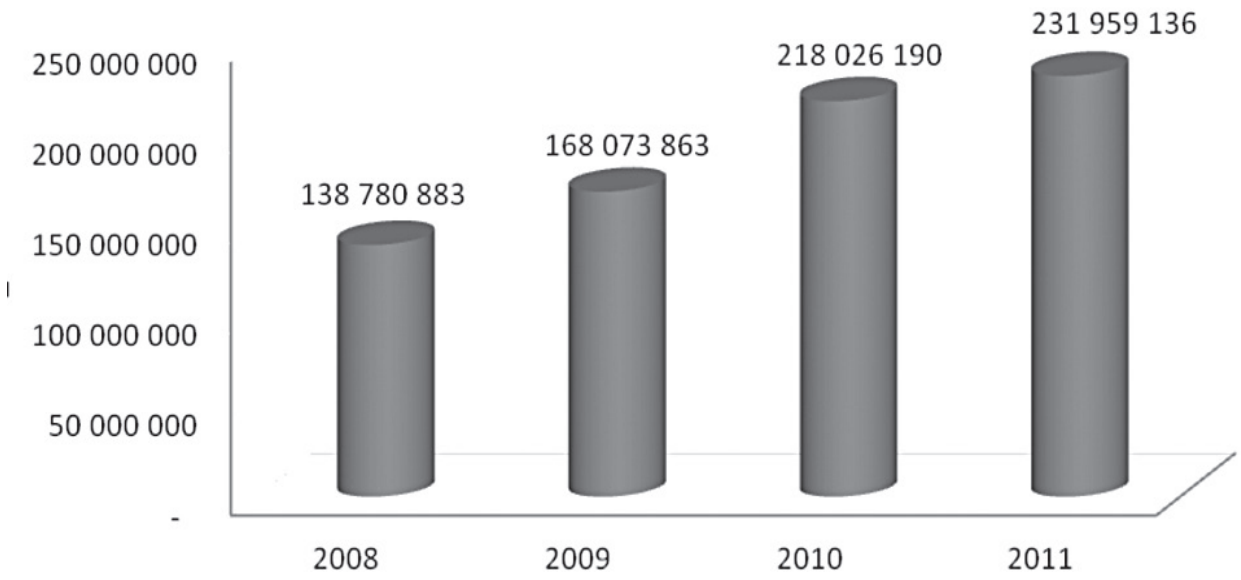

Figura 9. Facturación anual por el uso del teléfono en la UNA para los años 2008-20 I I.

Fuente: Sección de Financiero, ICE y UNA Campus Sostenible 2009-20 I 2.

Nota: La factura mensual por funcionario ronda los 5700 colones.

Cuadro 3. Indicadores de consumo de combustibles

\begin{tabular}{|c|c|c|c|c|c|}
\hline Consumo de combustible (diesel) & \multicolumn{4}{|c|}{ Año } \\
\hline Aspecto & 2008 & 2009 & 2010 & 2011 \\
\hline Monto $(\not)$ & 159137400 & 160031104 & 168129038 & 199404898 \\
\hline$L$ consumidos & 281819 & 360432 & 362684 & 332645 \\
\hline
\end{tabular}

Nota: Para el año 201 I la facturación del consumo de combustibles ascendió a 199698258 colones según la Sección Financiera y para el 2012, con la campaña "Únase al ahorro", se pretende reducir la facturación en un 2\% (aprox. 4 millones). Fuente: Sección de Transportes, Sección Financiera y UNA Campus Sostenible 2009-20I2.

\section{Resultados y discusión}

El consumo eléctrico de la UNA asciende a los

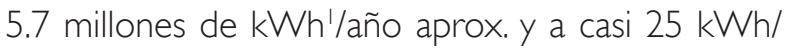
mes/persona. De 2008 a 2011 , la UNA presenta un consumo eléctrico de comportamiento convexo (Figura 3), donde en 2008 se consumieron 5.7 millones de $\mathrm{kWh}$, disminuyendo hasta 5.3 millones de $\mathrm{kWh}$ en 2010 e incrementándose en 2011 hasta 5.7 millones de $\mathrm{kWh}$ nuevamente. Este repunte en el consumo puede deberse al crecimiento de la población universitaria, al incremento de solicitudes de servicios o al aumento de locales de alquiler para oficinas, pero como aspecto a analizar, por las malas prácticas de utilización tanto de luminarias como de aires acondicionados (temperaturas de uso por

kWh: Kilowatt hora. debajo de $23^{\circ} \mathrm{C}$ ) y de equipos que quedan encendidos innecesariamente en tiempos de ocio.

El consumo de agua en la UNA ha tenido dos situaciones particulares en el tiempo. Una donde el consumo pasó de 183000 metros cúbicos en 2008 hasta 220000 metros cúbicos en 2009 (cuadro 2). Esto fue provocado esencialmente por el incremento de la infraestructura de los campus y el consecuente aumento del número de servicios necesarios. Esta situación se agravó para 2010 (cuadro 2), cuando se dio un aumento desproporcionado en el consumo, el cual ascendió a 327000 metros cúbicos y que se verificó en varias instancias: ESPH y Sección de Mantenimiento-UNA. La situación se dio cuando en 201 I el consumo de agua se mantuvo prácticamente igual al de 2010 (329 000 metros cúbicos). 
El consumo de combustible fósil en la UNA se puede analizar desde tres aspectos: Flota vehicular, plantas eléctricas, sodas y comedores en toda la institución y viajes aéreos por parte de estudiantes, invitados y profesores. En este estudio se analizará el aspecto de la flotilla vehicular y las plantas eléctricas. El consumo de combustibles por parte de la flotilla vehicular y plantas eléctricas sobrepasa los 330000 litros/año, utilizados en giras de investigación y académicas y diesel para las plantas eléctricas de emergencias de los edificios. Se debe entender que los procesos propios de la UNA y sus diferentes sedes y estaciones alrededor del país, exigen que exista una programación de giras hacia estos centros y de estos hacia la Sede Central. Por otro lado, las plantas eléctricas exigen un consumo mínimo para su operación y mantenimiento.

En el cuadro 3 se expresa el comportamiento del consumo de combustible, tanto en la facturación en colones como expresado en litros.

En la UNA, específicamente para el teléfono se entiende que se poseen dos tipos de servicios: análogos y tecnología IP. La facturación del servicio de teléfono se cancela mensualmente $y$, según los estudios de la Sección Financiera, esta facturación ha ido en aumento desde 2008 hasta 201। de forma sostenida. Lo anterior se refleja en los controles de pago por la Sección Financiera (ver cuadro 4).

Cuadro 4. Indicadores de facturación por el uso del teléfono

\begin{tabular}{|c|c|}
\hline Año & Total facturado $(\not)$ \\
\hline 2008 & 138780883 \\
\hline 2009 & 168073863 \\
\hline 2010 & 218026190 \\
\hline 2011 & 231959136 \\
\hline
\end{tabular}

Nota: Para el año 20 I l la facturación por el uso del teléfono ascendió a ф231 959 | 36 según la Sección Financiera y para 20 I2, con la campaña "Únase al ahorro", se pretende reducir la facturación en un 5\% (aprox. I 1,6 millones). Fuente: Sección

Financiera, ICE y UNA Campus Sostenible 2009-20I2.

\section{Conclusiones}

Cuando observamos el comportamiento mensual de consumo eléctrico en la UNA en el periodo 2008-201 I, podemos inferir que tiene una tendencia usual, ya que en los meses de enero se da el con- sumo más bajo, lo cual se debe a que la Universidad se encuentra en un período de receso. Además, en los meses de marzo-abril se da un valle en el consumo debido a la época de Semana Santa, donde la Universidad entra en un período de vacaciones. Algo similar sucede en los meses de julio-agosto (receso institucional de I 5 días) y diciembre (receso institucional). Así entonces, los meses de mayor consumo eléctrico son febrero (ingreso a lecciones), junio (previo a salida a receso institucional de medio período) y setiembre-octubre (previo a salida a receso institucional de fin de año).

Por otro lado, se debe continuar con labores de capacitación y sensibilización por parte del Programa UNA Campus Sostenible, con el establecimiento de la campaña "Únase al ahorro", además de impartirse cursos de ecoeficiencia, fortalecimiento de programa de compras verdes dirigido a la adquisición de dispositivos y aparatos eléctricos en coordinación con la Sección de Proveeduría. Adicionalmente, para la construcción de futuras infraestructuras, se puede implementar el uso de criterios eficientes de iluminación y ventilación naturales (Área de Planeamiento Espacial), además del cambio de tecnología en toda la Universidad para el uso de artefactos eficientes en consumo eléctrico y eficiencia lumínica, como es el proyecto desarrollado en la Biblioteca Central y en las diferentes facultades de la UNA por la Sección de Mantenimiento.

Si tomamos en cuenta que una persona promedio en los hogares consume 75 kilowatt/hora/mes, y lo comparamos con el consumo per cápita por mes de la UNA, se puede determinar que este último es un tercio del consumo en los hogares. Se debe tener en cuenta que la población estudiantil está presente en los campus en tiempos reducidos y se puede decir que está "de paso", a excepción de las residencias estudiantiles, donde se presentan hábitos domiciliarios de consumo. Por otro lado, los funcionarios están aproximadamente 8 horas presenciales pero en diferentes turnos y que al menos son dos turnos.

El consumo elevado de agua se dio en la Sede Central, específicamente en el Campus Omar Dengo, ya que en otras sedes, centros y estaciones los consumos presentados son los usuales. Sí existe evidencia de la gran cantidad de nuevas construcciones y remodelaciones, además de una reactivación de los procesos de limpieza de los tanques de 
almacenamiento en los meses de enero y la etapa de riego en la época seca (enero-abril) aunado al aumento de la población universitaria. Esto último ratificado en el comportamiento mensual en los años del consumo de agua. El consumo de agua per cápita se sitúa entre los 30 litros diarios (2008) y los 46 litros diarios (201 I). Cabe resaltar que una persona en su vivienda consume alrededor de 165 litros diarios y una persona en la UNA no supera los 50 litros diarios.

Se debe mencionar que el éxito en la reducción de consumo de agua siempre estará determinado por el esfuerzo por mejorar las prácticas usuales a la hora de utilizar este recurso (cambio de cultura), ya sea con la presente campaña de ahorro ("Únase al ahorro"), además de la generación de políticas específicas, el mantenimiento de las tuberías y tanques de almacenamiento de agua y el aprovechamiento de las aguas de las plantas de tratamiento para riego de áreas verdes (utilización de sistemas re utilizadores), entre otras. Si disminuye el consumo, disminuiría también el volumen de la producción de aguas residuales que van a las plantas de tratamiento.

LA UNA, de 2008 a 201 I, presenta un consumo en litros de combustible de comportamiento cóncavo, donde en 2008 se consumieron 282000 litros aproximadamente, aumentando hasta unos 363000 litros en 2010 y disminuyendo en 2011 hasta 333000 litros. Esta disminución en el consumo puede deberse al mejoramiento en la eficiencia de las giras y a la sistematización informática de las solicitudes de giras, al mantenimiento al menos correctivo de los automotores, pero como un aspecto digno de analizar se deben mejorar las buenas prácticas de conducción, el mantenimiento preventivos de los automotores, la eficiencia de giras cortas, la producción de diesel a partir de subproductos como el aceite de cocina, la compra de automóviles con nuevas tecnologías como autos híbridos o eléctricos y el aumento de la rigurosidad para la obtención de la Licencia de conducción UNA.

Por otro lado, se debe continuar con labores de capacitación y sensibilización por parte del Programa UNA Campus Sostenible en coordinación con la Vicerrectoría de Desarrollo, con el establecimiento de la campaña "Únase al ahorro", además de gestionar cursos de eficiencia en la conducción.

En términos económicos, el gasto en combustibles en el tiempo no disminuye. Esto está explicado por el aumento de los precios del litro de combustibles año a año.

En el uso del teléfono se da un incremento significativo año a año, el cual puede explicarse por el crecimiento de la población, el incremento de solicitudes de servicios y el aumento de locales de alquiler para oficinas, pero como aspecto digno de analizar está el uso del teléfono para asuntos ajenos de la institución. Para lo anterior, se recomienda el establecimiento de medidas de control, tales como la creación de códigos individuales para los funcionarios y el ajuste de límites de tiempo por llamada a 4 minutos máximo, por ejemplo. Como aspecto singular, la UNA paga más por el uso del teléfono al mes que por el uso de combustibles (más de 230 millones de colones/año). Se debe mencionar que cuando se analiza el uso del teléfono por funcionario, se sabe que por mes se pagan alrededor de 5700 colones, y que además este indicador durante los años no cambia significativamente desde 2009.

\section{Bibliografía}

Instituto Nacional de Acueductos y Alcantarillados (2009-20 I2). Datos de consumo de la UNA en medidores de aguaFacturación mensual por medidor 2008-2011.

Compañía Nacional de Fuerza y Luz (2009-2012). Datos de consumo de la UNA en medidores de luz- Facturación mensual por medidor 2008-20I I.

Departamento Financiero de la UNA (2009-2012). Datos de facturación de medidores de luz, agua, combustibles y teléfono de la UNA 2008-201 l.

Departamento de Registro de la UNA (2009-20 I 2). Información de cantidad de estudiantes en la UNA 2008-201।.

Empresa de Servicios Públicos de Heredia (2009-2012). Datos de consumo de la UNA en medidores de agua y luzFacturación mensual por medidor 2008-2011.

Instituto Costarricense de Electricidad (2009-2012). Datos de consumo de la UNA en medidores de luz 2008-20 I I.

Ministerio de Ambiente, Energía y Telecomunicaciones (201 I). Guía para laActualización de los Planes de Gestión Ambiental acorde al Decreto Ejecutivo no. 36499-S-MINAET.

Programa UNA Campus Sostenible (2012). Campaña "Únase al ahorro".

Programa UNA Campus Sostenible (2009-20 I I2). Manejo de registro estadístico de consumos en los años 2008-20l I.

Recursos Humanos de la UNA (2009-20l0-201 I). Información de cantidad de funcionarios de la UNA 20082011.

Refinería Costarricense de Petróleo (2009-2012). Información de Histórico de Precios del diesel, Densidad del diesel 2008-2011.

Sección de Transportes de la UNA (2009-20 I2). Datos de vehículos, Giras, Consumo de combustibles de la UNA 2008-20II. 